differences in injury severity between stabbing and firearms, both types of violence have similar distribution in most demographic groups in distinction from 'unarmed brawling' and 'assault with an object'.

Conclusions Despite the connection to violence rates and stabbing, unemployment has no linear connection to the severity of violence-related injuries. Nevertheless, the correlation of stabbing trend with firearms trend, which, in turn, had strong correlation with injury severity trend, suggests that a non-linear relationship could be present.

\section{WHAT DEFINES THE TREND OF VIOLENCE-RELATED INJURIES?}

M Rozenfeld*, K Peleg Correspondence: Gertner Institute for Health Policy \& Epidemiology Research, Tel-Hashomer, Ramat Gan 52621, Israel

10.1136/ip.2010.029215.101

Background Previous studies established a clear connection between unemployment and violence rates trends, but little is known about influence of such factors on trends in severity of violence-related injuries. As the severity of those injuries is known to be connected to types of violence, this study will look for correlation between the trends of all abovementioned factors.

Methods A retrospective study based on the National Trauma Registry data on patients hospitalised between 1 January 1998 and 31 December 2006 due to trauma. It consisted of 167142 , patient records of which 9839 (5.9\%) sustained violence-related injury. Descriptive statistics and bivariate analyses were used to characterise trends and injury patterns.

Results Unemployment trend had a significant correlation with violence rates and stabbing trends, but no connection to injury severity trend was found. Injury severity trend correlated positively with firearms trend and negatively with 'unarmed brawling' trend. Stabbing trend correlates negatively with firearms and 'assault with an object' trends. Despite polar 